\title{
Numerical analysis of compressible fluid flow in a channel with sharp contractions
}

\author{
L. Škerget \& J. Ravnik \\ University of Maribor, Faculty of Mechanical Engineering, \\ Maribor, Slovenia
}

\begin{abstract}
The problem of unsteady fluid flow in a channel with a sharp contraction is studied numerically. An incompressible and full compressible Navier-Stokes set of equations is considered. The thermal energy equation is written in its most general form including the Rayleigh and reversible expansion rate terms. Flows for different Reynolds number values are studied in the context of unsteadiness of the flow. The influence of the additional nonlinearity due to compressibility of the fluid, dissipation and reversible rate of work are analyzed. Also, their influence on the stability of the flow is considered.

The boundary element numerical model is used, with the velocity vorticity formulation of the Navier-Stokes equations. The pressure field is evaluated from the pressure Poisson equation. Material properties are taken to be for the ideal fluid (air), and assumed to be pressure and temperature dependent.
\end{abstract}

\section{Introduction}

There are a large variety of forced-convection processes for gases flowing in closed conduits which can be reasonably approximated as constant pressure processes. In this study we examine the thermal energy equation for a flow which is neither constant density nor constant pressure. Here we consider the unsteadiness of compressible viscous flow in channels with sharp contractions. Due to sharp discontinuity in the channel geometry the thermal energy equation is written in its expanded form with the terms such as the rate of reversible work and the rate of irreversible or dissipation work. The coupled momentum and thermal energy transport equations, specially due to mentioned rate of work terms, drastically increased 
the nonlinearity of the governing set of equations, and as a consequence decreased the stability of the numerical algorithm.

The unsteadiness of force convection flow are considered in a channel of aspect ratio $A=H / L=8$, with height $H$ and length $L$, with one and two sharp contractions. The flow behavior is computed for the Reynolds number values $R e=$ 20,200 and 2000. For the Reynolds number value $R e=20$ the steady flow exists, while for the Reynolds number values $R e=200$ and 2000 the flow is unsteady and oscillating with the transition to turbulent flow situation.

\section{Governing equations for the primitive variables formulation}

The field functions of interest are velocity vector field $v_{i}$, scalar pressure field $p$, temperature field $T$ and the field of mass density $\rho$, so that the mass, momentum and energy equations are given by the following set of nonlinear equations

$$
\begin{gathered}
\frac{\partial v_{j}}{\partial x_{j}}=\mathcal{D}=-\frac{1}{\rho} \frac{D \rho}{D t} \\
\rho \frac{D v_{i}}{D t}=-e_{i j k} \frac{\partial \eta \omega_{k}}{\partial x_{j}}+2 e_{i j k} \frac{\partial \eta}{\partial x_{j}} \omega_{k}+2 \frac{\partial \eta}{\partial x_{j}} \frac{\partial v_{i}}{\partial x_{j}}+\frac{4}{3} \frac{\partial \eta \mathcal{D}}{\partial x_{i}}-2 \mathcal{D} \frac{\partial \eta}{\partial x_{i}}-\frac{\partial p}{\partial x_{i}}+\rho g_{i}, \\
c \frac{D T}{D t}=\frac{\partial}{\partial x_{j}}\left(k \frac{\partial T}{\partial x_{j}}\right)+\beta T \frac{D p}{D t}+\Phi
\end{gathered}
$$

in the Cartesian frame $x_{i}$, where $c$ denotes changeable isobaric specific heat capacity per unit volume, $c=c_{p} \rho, t$ is time, $g_{i}$ is gravitational acceleration vector, while $\beta$ is a volume coefficient of thermal expansion and $\Phi$ is the Rayleigh viscous dissipation function. Because of the analytical reasons to develop the velocity-vorticity formulation of the governing equations, the momentum equation is given in the second extended form. The following forms of the linear constitutive models for compressible viscous shear fluid are considered, such as the Newton and Fourier law of momentum and thermal energy diffusion

$$
\tau_{i j}=2 \eta \dot{\varepsilon}_{i j}-\frac{2}{3} \eta \mathcal{D} \delta_{i j}, \quad q_{i}=-k \frac{\partial T}{\partial x_{i}},
$$

where $\mathcal{D}=\operatorname{div} \vec{v}=\dot{\varepsilon}_{i i}$ represents the divergence of the velocity field, and the Rayleigh dissipation function may be stated as

$$
\Phi=\tau_{i j} \frac{\partial v_{i}}{\partial x_{j}}=\eta\left(\frac{\partial v_{i}}{\partial x_{j}} \frac{\partial v_{i}}{\partial x_{j}}+\frac{\partial v_{j}}{\partial x_{i}} \frac{\partial v_{j}}{\partial x_{i}}\right)-\frac{2}{3} \eta \mathcal{D}^{2} .
$$

Representing the material properties of the fluid the dynamic viscosity $\eta$, heat conductivity $k$, the specific heat per unit volume $c$, and the mass density $\rho$, respectively, as a sum of a constant and variable part, e.g. $\eta=\eta_{o}+\widetilde{\eta}, k=k_{o}+\widetilde{k}$, $c=c_{o}+\widetilde{c}$, and $\rho=\rho_{o}+\widetilde{\rho}$, the momentum and energy eqs. (2) and (3) may be 
written in analogy to the basic conservation equations formulated for the constant material properties

$$
\begin{gathered}
\frac{D \vec{v}}{D t}=-\nu_{o} \vec{\nabla} \times \vec{\omega}-\frac{1}{\rho_{o}} \vec{\nabla} p+\frac{\rho}{\rho_{o}} \vec{g}+\frac{1}{\rho_{o}} \vec{f}^{m}, \\
\frac{D T}{D t}=a_{o} \triangle T+\frac{S^{m}}{c_{o}},
\end{gathered}
$$

where the pseudo body force term $\vec{f}^{m}$ and pseudo heat source term $S^{m}$ are introduced into the momentum eq. (6) and into energy eq. (7) respectively, capturing the variable material property effects, and the nonlinear effects due to rate of reversible and irreversible work, and given by expressions, e.g. for plane flow problems

$$
f_{i}^{m}=-e_{i j} \frac{\partial \widetilde{\eta} \omega}{\partial x_{j}}+2 e_{i j} \frac{\partial \widetilde{\eta}}{\partial x_{j}} \omega+2 \frac{\partial \eta}{\partial x_{j}} \frac{\partial v_{i}}{\partial x_{j}}+\frac{4}{3} \frac{\partial \eta \mathcal{D}}{\partial x_{i}}-2 \mathcal{D} \frac{\partial \eta}{\partial x_{i}}-\widetilde{\rho} a_{i},
$$

while the pseudo heat source term is given by an expression

$$
S^{m}=\vec{\nabla}(\widetilde{k} \vec{\nabla} T)-\widetilde{c} \frac{D T}{D t}+\beta T \frac{D p}{D t}+\Phi,
$$

in which the kinematic viscosity is $\nu_{o}=\eta_{o} / \rho_{o}$, the heat diffusivity $a_{o}=k_{o} / c_{o}$ and the inertia acceleration vector is $\vec{a}=D \vec{v} / D t$.

\section{Governing equations for the velocity-vorticity formulation}

The kinematics of the flow motion may be obtained by applying the curl operator to vorticity definition [2]:

$$
\vec{\nabla} \times \vec{\omega}=\vec{\nabla} \times(\vec{\nabla} \times \vec{v})=\vec{\nabla}(\vec{\nabla} \cdot \vec{v})-\Delta \vec{v},
$$

and by using the continuity eq. (1), the following vector elliptic Poisson equation for the velocity vector is derived

$$
\triangle \vec{v}+\vec{\nabla} \times \vec{\omega}-\vec{\nabla} \mathcal{D}=0
$$

The kinetics of the flow representing by the vorticity transport equation is obtained by applying the curl differential operator to the both sides of eq. (6), rendering the following statement for the two-dimensional plane flow written in Cartesian tensor notation form as

$$
\frac{\partial \omega}{\partial t}+\frac{\partial v_{j} \omega}{\partial x_{j}}=\nu_{o} \frac{\partial^{2} \omega}{\partial x_{j} \partial x_{j}}-\frac{1}{\rho_{o}} e_{i j} \frac{\partial \rho g_{i}}{\partial x_{j}}-\frac{1}{\rho_{o}} e_{i j} \frac{\partial f_{i}^{m}}{\partial x_{j}} .
$$

To derive the pressure equation, depending on known velocity field, vorticity field and material functions, the divergence of momentum equation should be calculated, resulting in the elliptic Poisson pressure equation

$$
\triangle p-\vec{\nabla} \cdot \overrightarrow{f_{p}}=0
$$


where the pressure force term $\vec{f}_{p}$ is for the planar flow cases

$$
\frac{\partial p}{\partial x_{i}}=f_{p i}=-\eta_{o} e_{i j} \frac{\partial \omega}{\partial x_{j}}-\rho_{o} a_{i}+\rho g_{i}+f_{i}^{m} .
$$

The Neumann boundary conditions for pressure equation may be determined for the whole solution domain and the following relation is valid

$$
\frac{\partial p}{\partial n}=\overrightarrow{f_{p}} \cdot \vec{n} \quad \text { on } \quad \Gamma .
$$

Due to the variable material property terms, and rate of reversible and irreversible work acting as additional temperature, pressure and velocity field dependent source terms, the vorticity, thermal energy, and pressure equations are coupled, making the numerical solution procedure of this highly nonlinear coupled set of equations very severe. Already, the vorticity transport equation as itself is highly nonlinear partial differential equation due to the inherent nonlinearity caused by the compatibility and restriction conditions among velocity, vorticity and dilatation fields. The dilatation and the vortical part of the flow, $\mathcal{D}$ and $\vec{\omega}$ field functions respectively, and all other nonlinear terms have to be underrelaxed to achieve convergence of the numerical solution procedure.

\section{Boundary-domain integral equations}

The singular boundary-domain integral representation for the velocity vector can be formulated by using the Green theorems for scalar functions or weighting residuals technique rendering the following vector integral formulation [1-10]), e.g. the plane two-dimensional kinematics is given by two scalar equations as follows

$$
c(\xi) v_{i}(\xi)+\int_{\Gamma} v_{i} q^{\star} d \Gamma=e_{i j} \int_{\Gamma} v_{j} q_{t}^{\star} d \Gamma-e_{i j} \int_{\Omega} \omega q_{j}^{\star} d \Omega+\int_{\Omega} \mathcal{D} q_{i}^{\star} d \Omega,
$$

or in the form of integral vector formulation for the general flow situation

$$
c(\xi) \vec{v}(\xi)+\int_{\Gamma} \vec{v} q^{\star} d \Gamma=\int_{\Gamma}\left(\vec{q}^{\star} \times \vec{n}\right) \times \vec{v} d \Gamma+\int_{\Omega} \vec{\omega} \times \vec{q}^{\star} d \Omega+\int_{\Omega} \mathcal{D} \vec{q}^{\star} d \Omega .
$$

Considering the vorticity kinetics in an integral representation one has to take into account parabolic diffusion convection character of the vorticity transport equation. With the use of the linear parabolic diffusion differential operator, the following integral formulation can be written

$$
\begin{gathered}
c(\xi) \omega\left(\xi, t_{F}\right)+\int_{\Gamma} \omega Q^{\star} d \Gamma=\frac{1}{\eta_{o}} \int_{\Gamma}\left(\eta_{o} \frac{\partial \omega}{\partial n}-\rho_{o} v_{n} \omega+\rho g_{t}+f_{t}^{m}\right) U^{\star} d \Gamma \\
+\frac{1}{\eta_{o}} \int_{\Omega}\left(\rho_{o} v_{j} \omega+\rho e_{i j} g_{i}+e_{i j} f_{i}^{m}\right) Q_{j}^{\star} d \Omega+\int_{\Omega} \omega_{F-1} u_{F-1}^{\star} d \Omega,
\end{gathered}
$$

in which a constant variation of all field and material functions within the individual time increment $\Delta t=t_{F}-t_{F-1}$ is assumed [11], e.g. the values at $t=t_{F}$ are 
considered for each time step, and where $v_{n}, g_{t}$ and $f_{t}^{m}$ are the normal velocity, and the tangential gravity and nonlinear material source components, respectively, e.g. $v_{n}=\vec{v} \cdot \vec{n}, g_{t}=\vec{g} \cdot \vec{t}=-e_{i j} g_{i} n_{j}$ and $f_{t}^{m}=\overrightarrow{f^{m}} \cdot \vec{t}=-e_{i j} f_{i}^{m} n_{j}$.

The pressure eq. (13) is an elliptic Poisson equation, and therefore employing again the linear elliptic Laplace differential operator the following form of the pressure integral equation is obtained

$$
c(\xi) p(\xi)+\int_{\Gamma} p q^{\star} d \Gamma=\int_{\Omega} f_{p i} q_{i}^{\star} d \Omega,
$$

where the vector $\vec{f}_{p}$ is given by eq. (14).

The integral representation of the nonlinear heat energy transport equation is derived considering the linear parabolic diffusion differential operator, therefore the following integral representation for the thermal energy kinetics can be evaluated

$$
\begin{gathered}
c(\xi) T\left(\xi, t_{F}\right)+\int_{\Gamma} T Q^{\star} d \Gamma=\frac{1}{k_{o}} \int_{\Gamma}\left(k \frac{\partial T}{\partial n}-c v_{n} T\right) U^{\star} d \Gamma \\
-\frac{1}{k_{o}} \int_{\Omega}\left(\widetilde{k} \frac{\partial T}{\partial x_{j}}-c v_{j} T\right) Q_{j}^{\star} d \Omega \\
+\frac{1}{k_{o}} \int_{\Omega}\left(T v_{j} \frac{\partial c}{\partial x_{j}}+c T \mathcal{D}-\widetilde{c} \frac{\partial T}{\partial t}+\beta T \frac{D p}{D t}+\Phi\right) U^{\star} d \Omega+\int_{\Omega} T_{F-1} u_{F-1}^{\star} d \Omega .
\end{gathered}
$$

\section{Numerical example}

Incompressible and compressible fluid flow circumstances in channels with one or two contractions are studied. Very coarse mesh is applied first, consisting of 168 quadratic boundary elements and 888 quadratic internal cells with 3721 total nodes, thus the numerical simulation results are more or less of only qualitative value to show the applicability of the presented numerical algorithm. The solution domain is shown in Figure 1.

The ideal gas (air) is chosen as a working fluid with the inflow temperature $T_{o}=600 \mathrm{~K}$ and pressure $p_{o}=101325 \mathrm{~Pa}$, the conduit walls are adiabatic, while at the outflow the convective temperature boundary conditions are prescribed. The flow field at Reynolds number values $R e=20,200$, and 2000 are simulated. In presented cases the Pr number value is assumed to remain constant (0.71), while the temperature dependence of the viscosity is given by the Sutherland's model

$$
\frac{\eta(T)}{\eta^{*}}=\left(\frac{T}{T^{*}}\right)^{\frac{3}{2}} \frac{T^{*}+S}{T+S},
$$

and the heat conductivity is expressed as

$$
k(T)=\frac{\eta(T) c_{p}}{\operatorname{Pr}}
$$

with $T^{*}=273 K, S=110.5 K, \eta^{*}=1.68 \cdot 10^{-5} \mathrm{~kg} / \mathrm{m} / \mathrm{s}, c_{p}=\kappa R /(\kappa-1)$, $\kappa=1.4$ and $R=287.0 \mathrm{~J} / \mathrm{kgK}$. The influence of temperature on $c_{p}$ is neglected. 
184 Boundary Elements and Other Mesh Reduction Methods XXIX
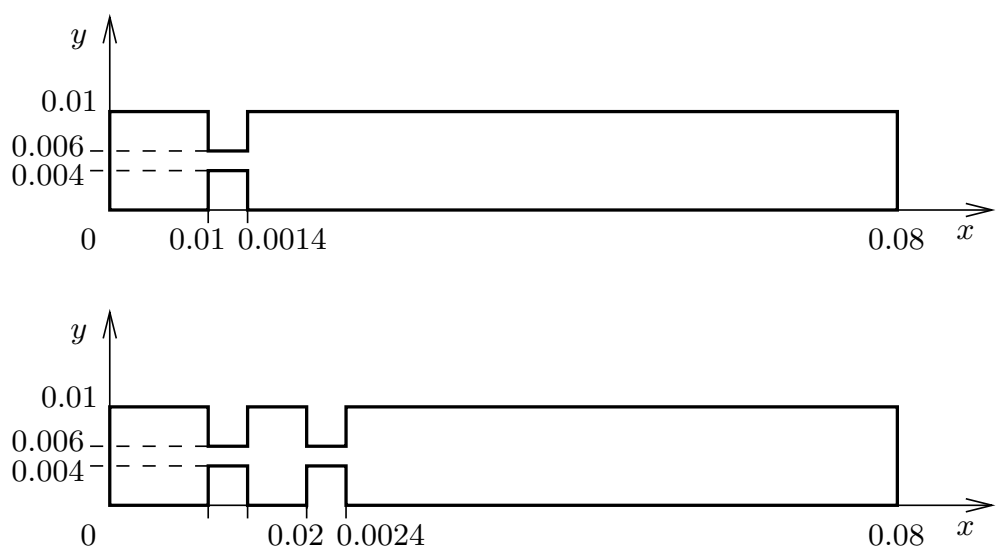

Figure 1: Numerical simulation flow domain: channel with one and two sharp contractions.

The steady state numerical simulation results for the velocity vector, pressure and temperature contours are presented in Figure 2 for $R e=20$ for the compressible coupled momentum energy transport.

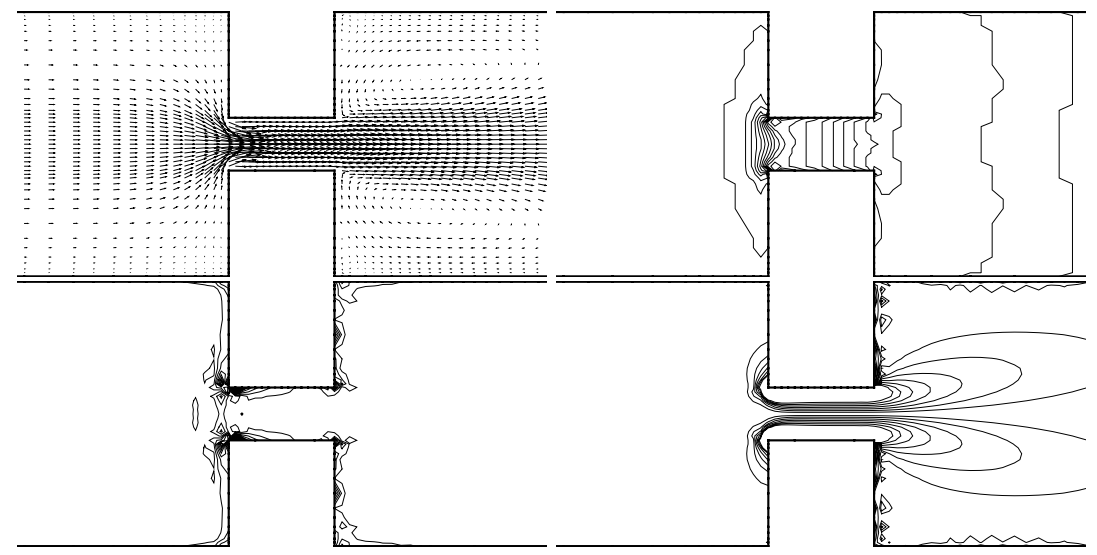

Figure 2: Numerical simulation results for $R e=20$ for the compressible coupled momentum energy transport: velocity vector, pressure, temperature and vorticity contours.

It is evident that the flow is basically incompressible and that all nonlinear effects, such as the irreversible and reversible rate of work, are negligible and localized only on the motion around sharp edges of the contraction. For the qualitative purposes, for this very course mesh, $T_{\min }=584 \mathrm{~K}$ and $T_{\max }=612 \mathrm{~K}$. 
The numerical solution results for the compressible and incompressible cases in general coincide.

For the $R e=200$ flow case the steady state do not exist and the flow oscillates with strong separation of the boundary layer. The unsteady state numerical simulation results for the compressible coupled momentum energy transport for the velocity vector, pressure and temperature contours are presented in Figure 3 for $R e=200$.
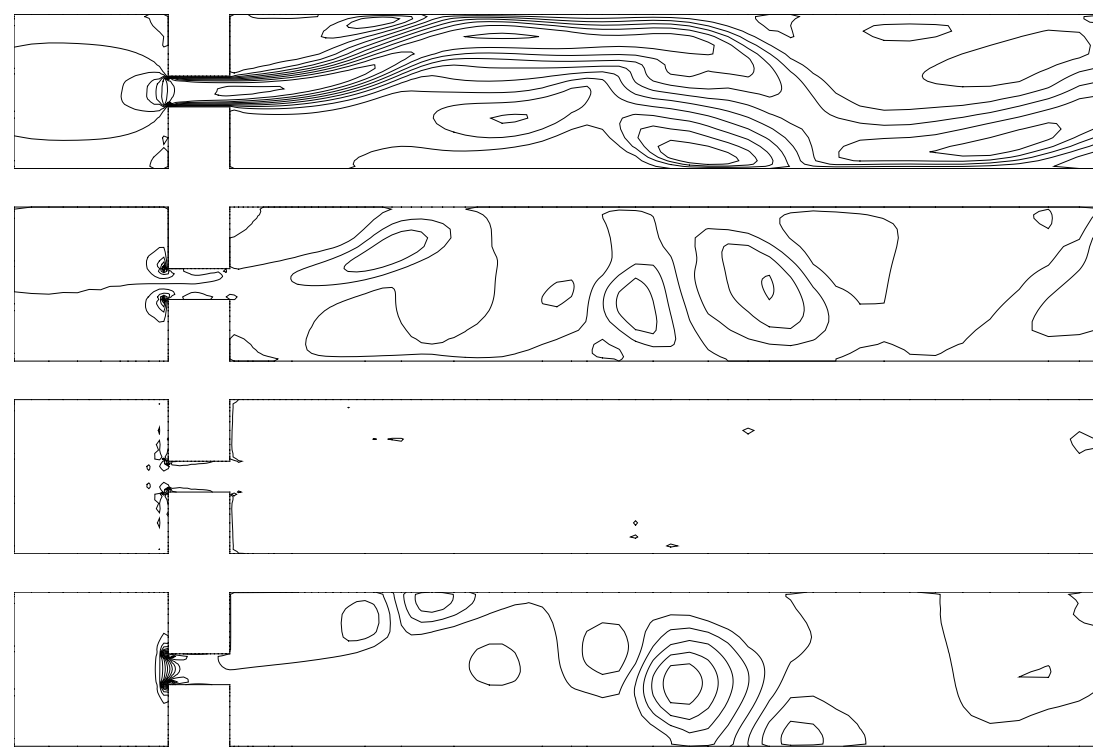

Figure 3: Numerical simulation results for $R e=200$ for the compressible coupled momentum energy transport: velocity vector $v_{x}, v_{y}$, temperature and pressure contours.

Again the compressibility and all other nonlinear effects in momentum and thermal energy processes are negligible in fluid flow domain and only localized on the areas around sharp edges of the contraction. For the qualitative purposes $T_{\min }=379 \mathrm{~K}$ and $T_{\max }=666 \mathrm{~K}$, thus the nonlinear effects are more severe but still very localized. The increased nonlinearity decrease the stability of the numerical procedure, very small time increment $\triangle t=10^{-4}$ has to be applied.

The flow field at Reynolds number value $R e=2000$ was simulated next with the time increment $\triangle t=10^{-5}$. Figure 4 shows the velocity contours, vorticity magnitude and pressure contours at a chosen time instant for the incompressible uncoupled transport case. Time traces of velocity components, vorticity and pressure a location on the centerline of the channel is plotted in Figure 5. 


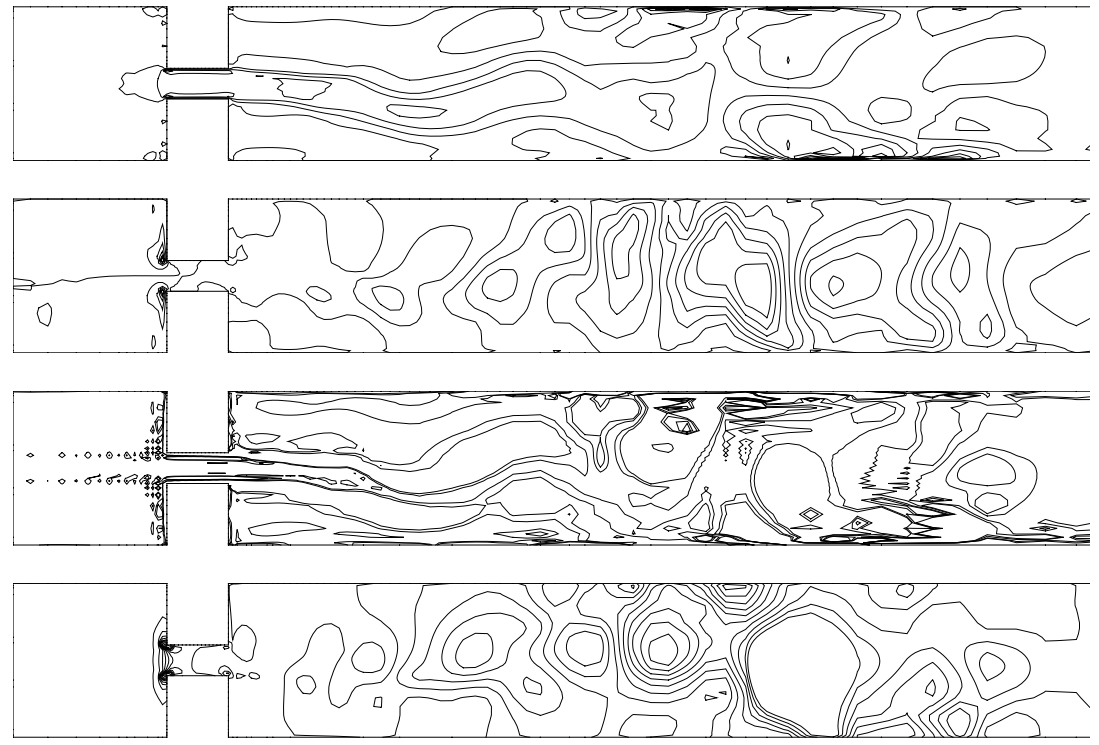

Figure 4: Numerical simulation results for $R e=2000$ for the incompressible uncoupled flow case: horizontal velocity, vertical velocity, vorticity and pressure contours a $t=4 \cdot 10^{-3}$.

\section{Conclusions}

In this work the boundary element integral approach to the solution of incompressible and compressible fluid motion in channels with sharp contractions is presented. The derived numerical model is characterized by decomposition of flow into its kinematic, vorticity kinetics, thermal energy kinetics, and pressure formulation a result of the velocity-vorticity formulation of the Navier-Stokes equation for a compressible fluid. The described numerical scheme leads to strong coupling between velocity, vorticity, temperature, pressure and mass density fields. The application of the elliptic Laplace and parabolic diffusion fundamental solutions in the derivation of integral representations ensures an accurate computation of the flow field variables.

The computed test examples confirm the applicability of BEM based numerical scheme also for a highly nonlinear transport phenomena, what compressible coupled momentum and thermal energy convection flow certainly is. The transient simulation results show development of the flow field with time caused by the compressible and viscous effects, as represented by the local expansion and vorticity field functions, respectively. The BEM mesh applied in the paper is appropriate to describe the compressible coupled momentum and thermal energy transport at Reynolds number values $R e=20$ and $R e=200$, but to coarse for transport phenomena at $R e=2000$. The nonlinearities in thermal energy equation are to severe, 

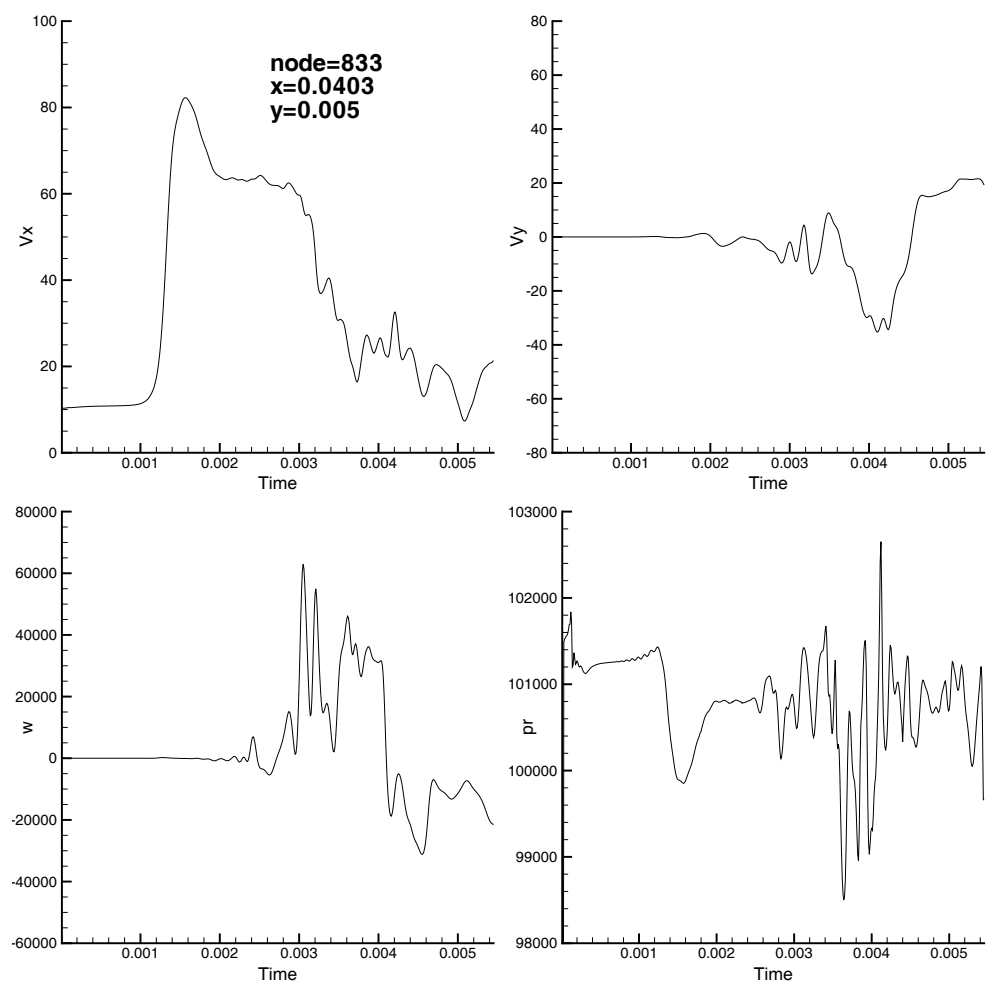

Figure 5: Numerical simulation results for $R e=2000$ for the incompressible uncoupled flow case: time traces of horizontal velocity, vertical velocity, vorticity and pressure at $x=0.0403, y=0.005$.

for the mesh applied, and only compressible and incompressible uncoupled cases are simulated successfully at $R e=2000$.

Results for the case of two contractions as well as further numerical analyses of the transition from periodic to turbulent flow circumstances will be presented at the conference.

\section{References}

[1] Škerget, L., Hriberšek, M., Kuhn, G. (1999) : Computational fluid dynamics by boundary-domain integral method. Int. J. Numer. Meth. Engng., Vol. 46, pp. 1291-1311.

[2] Škerget, L., Hriberšek, M., Žunič, Z. (2003) : Natural convection flows in complex cavities by BEM. International Journal of Numerical Methods for Heat \& Fluid Flow, Vol. 13, No. 6.

[3] Wu, J.C., Thompson, J.F. (1973) : Numerical solution of time dependent 
incompressible Navier-Stokes equations using an integro-differential formulation. Computers and Fluids, Vol. 1, pp. 197-215.

[4] Rizk, Y.M. (1980) : An integral representation approach for time dependent viscous flow. PhD Thesis, Georgia Institute of Technology.

[5] Wu, J.C. (1982) : Problems of general viscous fluid flow. Developments in BEM, Vol. 2, Ch. 2, Elsevier Appl. Sci. Publ., London and N.Y.

[6] Wu, J.C., Rizk, Y.M., Sankar, N.L. (1984) : Problems of time-dependent Navier-Stokes flow. Developments in BEM, Vol. 3, Ch. 6, Elsevier Appl. Sci. Publ., London and N.Y.

[7] Skerget, L., Alujevic, A., Brebbia, C.A., Kuhn, G. (1989) : Natural and forced convection simulation using the velocity-vorticity approach. Topics in Boundary element Research, Vol. 5, pp. 49-86, Springer-Verlag, Berlin.

[8] Škerget, L., Hriberšek, M., Kuhn, G. (1999) : Computational Fluid Dynamics by Boundary Domain Integral Method; Int. J. Num. Meth. Eng., Vol. 46, pp. 1291-1311.

[9] Wrobel. L.C. (2002) : The boundary element method. Vol. 1, Applications in Thermo-fluids and acoustics. Wiley.

[10] Skerget, L., Jecl, R. (2004) : Compressible fluid dynamics in porous media by the boundary element method. Emerging Technologies and Techniques in Porous Media, Eds. Ingham, D.B., Bejan, A., Mamut, E.. Pop, I. NATO Science Series, Vol. 134, Ch. 6, Kluwer Academic Publ., Dordrecht, Boston, London.

[11] Škerget, L., Samec, N. (2005) : BEM for the two-dimensional plane compressible fluid dynamics; Eng. Analysis with Boundary Elements, Vol. 29, pp. 41-57. 